\title{
Nonplanar periodic solutions for spatial restricted 3-body and 4-body problems
}

\section{Xiaoxiao Zhao ${ }^{1 *}$ and Shiqing Zhang ${ }^{2}$}

\section{${ }^{*}$ Correspondence:}

zxgg2007@163.com

'School of Mathematics and

Statistics, Henan University of

Science and Technology, Luoyang, 471023, People's Republic of China

Full list of author information is

available at the end of the article

\begin{abstract}
In this paper, by using variational methods, we study the existence of nonplanar periodic solutions for the following spatial restricted 3-body and 4-body problems: for $N=2$ or $3, N$ mass points with positive masses $m_{1}, \ldots, m_{N}$ move in a central configuration (for $N=2$, two bodies are in a Euler configuration; for $N=3$, three bodies are in a Lagrange configuration), and they move in the plane of $N$ circular obits; the $N+1$ th mass point, called the zero mass point, moves on the perpendicular axis passing through the center of the masses.
\end{abstract}

MSC: $34 \mathrm{C} 15 ; 34 \mathrm{C} 25 ; 58 \mathrm{E} 30$

Keywords: restricted 3-body problems; restricted 4-body problems; nonplanar periodic solutions; variational minimizers; the second variation

\section{Introduction and main results}

In this paper, we study the spatial circular restricted 3-body and 4-body problems. For $N=2$ or 3 , suppose $N$ mass points with positive masses $m_{1}, \ldots, m_{N}$ move in the plane of their circular orbits $q_{1}(t), \ldots, q_{N}(t)$, the radius $r_{1}, \ldots, r_{N}$ of orbits are all positive and the center of masses is at the origin; suppose the $N+1$ th mass point, called the zero mass point, does not influence the motion of the given $N$ mass points, and moves on the vertical axis of the plane for the first $N$ mass points, here the vertical axis passes through the center of masses.

It is known that $q_{1}(t), \ldots, q_{N}(t)(N=2$ or 3$)$ satisfy the Newtonian equations:

$$
m_{i} \ddot{q}_{i}=\frac{\partial U}{\partial q_{i}}, \quad i=1, \ldots, N
$$

where

$$
U=\sum_{1 \leq i<j \leq N} \frac{m_{i} m_{j}}{\left|q_{i}-q_{j}\right|}
$$

The orbit $q(t)=(0,0, z(t)) \in R^{3}$ for zero mass point is governed by the gravitational forces of $m_{1}, \ldots, m_{N}(N=2$ or 3$)$ and therefore it satisfies the following equation:

$$
\ddot{q}=\sum_{i=1}^{N} \frac{m_{i}\left(q_{i}-q\right)}{\left|q_{i}-q\right|^{3}}, \quad N=2 \text { or } 3 .
$$

(c) 2015 Zhao and Zhang. This article is distributed under the terms of the Creative Commons Attribution 4.0 International License (http://creativecommons.org/licenses/by/4.0/), which permits unrestricted use, distribution, and reproduction in any medium, provided you give appropriate credit to the original author(s) and the source, provide a link to the Creative Commons license, and indicate if changes were made. 
For $N \geq 2$, there are many papers concerned with the restricted $N$-body problem; see [1-6] and the references therein. In [7], Sitnikov considered the following model: two mass points of equal mass $m_{1}=m_{2}=\frac{1}{2}$ move in the plane of their elliptic orbits and the center of the masses is at rest, the third mass point which does not influence the motion of the first two moves on the line perpendicular to the plane containing the first two mass points and goes through the center of mass, and he used geometrical methods to prove the existence of the oscillatory parabolic orbit of

$$
\ddot{z}(t)=\frac{-z(t)}{\left(|z(t)|^{2}+|r(t)|^{2}\right)^{3 / 2}},
$$

where $r(t)=r(t+2 \pi)>0$ is the distance from the center of mass to one of the first two mass points. McGehee [4] used stable and unstable manifolds to study the homoclinic orbits (parabolic orbits) of (1.4). In [5], Mathlouthi studied the periodic solutions for the spatial circular restricted 3-body problems by minimax variational methods. Li et al. [1] used Jacobi's necessary conditions for the variational minimizers to study the existence of nonplanar periodic solutions for spatial restricted $N+1$-body problems with a zero mass moving on the vertical axis of the plane for $N$ equal masses.

In this article, we study the spatial circular restricted 3-body and 4-body problems with a zero mass point moving on the perpendicular axis of the circular orbits plane for given masses $m_{1}, \ldots, m_{N}(N=2$ or 3$)$ in their respective central configuration.

Define

$$
W^{1,2}(R / T Z, R)=\left\{u(t) \mid u(t), u^{\prime}(t) \in L^{2}(R, R), u(t+T)=u(t)\right\} .
$$

The inner product and the norm of $W^{1,2}(R / T Z, R)$ are

$$
\begin{aligned}
& \langle u, v\rangle=\int_{0}^{T}\left(u v+u^{\prime} \cdot v^{\prime}\right) d t \\
& \|u\|=\left[\int_{0}^{T}|u|^{2} d t\right]^{\frac{1}{2}}+\left[\int_{0}^{T}\left|u^{\prime}\right|^{2} d t\right]^{\frac{1}{2}} .
\end{aligned}
$$

We consider the Lagrangian functional of (1.3)

$$
\begin{aligned}
f(q) & =\int_{0}^{T}\left[\frac{1}{2}|\dot{q}|^{2}+\sum_{i=1}^{N} \frac{m_{i}}{\left|q-q_{i}\right|}\right] d t \\
& =\int_{0}^{T}\left[\frac{1}{2}\left|z^{\prime}\right|^{2}+\frac{m_{1}}{\sqrt{r_{1}^{2}+z^{2}}}+\cdots+\frac{m_{N}}{\sqrt{r_{N}^{2}+z^{2}}}\right] d t \triangleq f(z), \quad N=2 \text { or } 3
\end{aligned}
$$

on $\Lambda_{j}, j=1,2$, where

$$
\Lambda_{1}=\left\{q(t)=(0,0, z(t)) \mid z(t) \in W^{1,2}(R / T Z, R), z\left(t+\frac{T}{2}\right)=-z(t)\right\}
$$

and

$$
\Lambda_{2}=\left\{q(t)=(0,0, z(t)) \mid z(t) \in W^{1,2}(R / T Z, R), z(-t)=-z(t)\right\} .
$$


Our main results are the following.

Theorem 1.1 For $N=2$, let the mass points of $m_{1}, m_{2}$ be in a Euler configuration, then the minimizer of $f(q)$ on the closure $\bar{\Lambda}_{i}$ of $\Lambda_{i}(i=1,2)$ exists and it is a nonplanar and noncollision periodic solution of (1.3).

Theorem 1.2 For $N=3$, let the mass points of $m_{1}, m_{2}, m_{3}$ be in a Lagrange configuration, then the minimizer of $f(q)$ on the closure $\bar{\Lambda}_{i}$ of $\Lambda_{i}(i=1,2)$ exists and it is a nonplanar and noncollision periodic solution of (1.3).

Remark 1.1 Obviously, the nonplanar periodic solutions we got in the above two theorems are collisionless.

\section{Preliminaries}

In this section, we will list some basic lemmas and inequality for proving our Theorems 1.1 and 1.2 .

Lemma 2.1 (Poincaré-Wirtinger inequality [8]) Let $q \in W^{1,2}\left(R / T Z, R^{K}\right)$ and $\int_{0}^{T} q(t) d t=$ 0 , then

$$
\int_{0}^{T}|q(t)|^{2} d t \leq \frac{T^{2}}{4 \pi^{2}} \int_{0}^{T}|\dot{q}(t)|^{2} d t
$$

Lemma 2.2 (Tonelli [3]) Let $X$ be a reflexive Banach space, $S$ be a weakly closed subset of $X, f: S \rightarrow R \cup+\infty$. If $f \not \equiv+\infty$ is weakly lower semi-continuous and coercive $(f(x) \rightarrow+\infty$ as $\|x\| \rightarrow+\infty)$, then $f$ attains its infimum on $S$.

Lemma 2.3 (Palais' symmetry principle [9]) Let $\sigma$ be an orthogonal representation of a finite or compact group $G, H$ be a real Hilbert space, $f: H \rightarrow R$ satisfies $f(\sigma \cdot x)=f(x)$, $\forall \sigma \in G, \forall x \in H$.

Set $F=\{x \in H \mid \sigma \cdot x=x, \forall \sigma \in G\}$. Then the critical point of $f$ in $F$ is also a critical point off in $H$.

Remark 2.1 By Palais' symmetry principle, we know that the critical point of $f(q)$ in $\bar{\Lambda}_{i}=$ $\Lambda_{i}(i=1,2)$ is a periodic solution of Newtonian equation (1.3).

Lemma 2.4 $f(q)$ in (1.7) attains its infimum on $\bar{\Lambda}_{i}=\Lambda_{i}(i=1,2)$.

Proof By using Lemma 2.1, for $\forall z \in \Lambda_{i}, i=1,2$, the equivalent norm of $(1.6)$ in $\Lambda_{i}(i=1,2)$ is

$$
\|z\| \cong\left[\int_{0}^{T}\left|z^{\prime}\right|^{2} d t\right]^{\frac{1}{2}} .
$$

Hence by the definitions of $f(q)$, it is easy to see that $f$ is $C^{1}$ and coercive on $\Lambda_{i}(i=1,2)$. In order to get Lemma 2.4 , we only need to prove that $f$ is weakly lower semi-continuous on $\Lambda_{i}(i=1,2)$. In fact, for $\forall z_{n} \in \Lambda_{i}$, if $z_{n} \rightarrow z$ weakly, by compact embedding theorem, we 
have uniform convergence:

$$
\max _{0 \leq t \leq T}\left|z_{n}(t)-z(t)\right| \rightarrow 0, \quad n \rightarrow \infty,
$$

which implies

$$
\begin{aligned}
& \int_{0}^{T} \frac{m_{1}}{\sqrt{r_{1}^{2}+z_{n}^{2}}}+\cdots+\frac{m_{N}}{\sqrt{r_{N}^{2}+z_{n}^{2}}} d t \\
& \rightarrow \int_{0}^{T} \frac{m_{1}}{\sqrt{r_{1}^{2}+z^{2}}}+\cdots+\frac{m_{N}}{\sqrt{r_{N}^{2}+z^{2}}} d t, \quad N=2 \text { or } 3 .
\end{aligned}
$$

It is well known that the norm and its square are weakly lower semi-continuous. Therefore, combined with (2.4), we obtain

$$
\liminf _{n \rightarrow \infty} f\left(z_{n}\right) \geq f(z),
$$

that is, $f$ is weakly lower semi-continuous on $\Lambda_{i}(i=1,2)$. By Lemma 2.2, we can see that $f(q)$ in (1.7) attains its infimum on $\bar{\Lambda}_{i}=\Lambda_{i}(i=1,2)$.

\section{Proof of Theorem 1.1}

In this section, we consider the spatial circular restricted 3-body problem with a zero mass point moving on the vertical axis of the moving plane for two mass points with arbitrary given positive masses $m_{1}, m_{2}$ in a Euler configuration. Suppose the planar circular orbits are

$$
\begin{aligned}
& q_{1}(t)=\left(r_{1} \cos \frac{2 \pi}{T} t, r_{1} \sin \frac{2 \pi}{T} t, 0\right), \\
& q_{2}(t)=\left(-r_{2} \cos \frac{2 \pi}{T} t,-r_{2} \sin \frac{2 \pi}{T} t, 0\right) ;
\end{aligned}
$$

here the radii $r_{1}, r_{2}$ are positive constants depending on $m_{i}(i=1,2)$ and $T$ (see Lemma 3.1). We also assume that

$$
m_{1} q_{1}(t)+m_{2} q_{2}(t)=0 .
$$

We consider the Lagrangian functional of (1.3)

$$
\begin{aligned}
f(q) & =\int_{0}^{T}\left[\frac{1}{2}|\dot{q}|^{2}+\frac{m_{1}}{\left|q-q_{1}\right|}+\frac{m_{2}}{\left|q-q_{2}\right|}\right] d t \\
& =\int_{0}^{T}\left[\frac{1}{2}\left|z^{\prime}\right|^{2}+\frac{m_{1}}{\sqrt{r_{1}^{2}+z^{2}}}+\frac{m_{2}}{\sqrt{r_{2}^{2}+z^{2}}}\right] d t \triangleq f(z)
\end{aligned}
$$

on $\Lambda_{i}, i=1,2$.

Lemma 3.1 The radii $r_{1}, r_{2}$ of the planar circular orbits for the masses $m_{1}, m_{2}$ are

$$
r_{1}=\left(\frac{T}{2 \pi\left(m_{1}+m_{2}\right)}\right)^{\frac{2}{3}} m_{2}, \quad r_{2}=\left(\frac{T}{2 \pi\left(m_{1}+m_{2}\right)}\right)^{\frac{2}{3}} m_{1} .
$$


Proof Substituting (3.1), (3.2) into (3.3), it is easy to get

$$
r_{2}=\frac{m_{1}}{m_{2}} r_{1}
$$

It follows from (1.1) and (1.2) that

$$
\ddot{q}_{1}=m_{2} \frac{q_{2}-q_{1}}{\left|q_{2}-q_{1}\right|^{3}} .
$$

Then by (3.1), (3.2), and (3.5), we have

$$
-\frac{4 \pi^{2}}{T^{2}} q_{1}=m_{2} \frac{\left(-\frac{m_{1}}{m_{2}}-1\right) q_{1}}{r_{1}^{3}\left|-\frac{m_{1}}{m_{2}}-1\right|^{3}}
$$

which implies

$$
r_{1}=\left(\frac{T}{2 \pi\left(m_{1}+m_{2}\right)}\right)^{\frac{2}{3}} m_{2}
$$

Hence by (3.5), we obtain

$$
r_{2}=\left(\frac{T}{2 \pi\left(m_{1}+m_{2}\right)}\right)^{\frac{2}{3}} m_{1}
$$

Proof of Theorem 1.1 Clearly, $q(t)=(0,0,0)$ is a critical point of $f(q)$ on $\bar{\Lambda}_{i}=\Lambda_{i}(i=1,2)$. The second variation of (3.4) in the sphere neighborhood of $q(t)=(0,0,0)$ (coordinate origin $O$ ) is given by

$$
\delta^{2} f(O, \varphi)=\int_{0}^{T}\left(\varphi_{3}^{\prime 2}-\left(\frac{m_{1}}{r_{1}^{3}}+\frac{m_{2}}{r_{2}^{3}}\right) \varphi_{3}^{2}\right) d t, \quad \forall \varphi=\left(0,0, \varphi_{3}\right) \in \Lambda_{i}, i=1,2
$$

Let

$$
\tilde{\varphi}(t)=\left(0,0, \sin \frac{2 \pi}{T} t\right)
$$

Obviously, $\tilde{\varphi} \in \Lambda_{i}(i=1,2)$ and

$$
\dot{\tilde{\varphi}}(t)=\left(0,0, \frac{2 \pi}{T} \cos \frac{2 \pi}{T} t\right) .
$$

Since

$$
m_{1}^{6}+m_{2}^{6} \geq 2 \sqrt{m_{1}^{6} \cdot m_{2}^{6}}=2 m_{1}^{3} m_{2}^{3}>m_{1}^{3} m_{2}^{3}
$$

it is easy to see that

$$
\begin{aligned}
\left(m_{1}^{4}+m_{2}^{4}\right)\left(m_{1}+m_{2}\right)^{2} & >m_{1}^{6}+m_{2}^{6} \\
& >m_{1}^{3} m_{2}^{3},
\end{aligned}
$$


which implies

$$
\frac{\sqrt{m_{1}^{3} m_{2}^{3}}}{\sqrt{m_{1}^{4}+m_{2}^{4}}\left(m_{1}+m_{2}\right)}<1,
$$

that is

$$
\sqrt{\frac{m_{1}^{4}+m_{2}^{4}}{m_{1}^{3} m_{2}^{3}}}\left(m_{1}+m_{2}\right)>1 .
$$

It follows from (3.8), (3.9), and (3.16) that

$$
\begin{aligned}
\sqrt{\frac{m_{1}}{r_{1}^{3}}+\frac{m_{2}}{r_{2}^{3}}} & =\sqrt{\frac{m_{1}^{4}+m_{2}^{4}}{m_{1}^{3} m_{2}^{3}}}\left(m_{1}+m_{2}\right) \cdot \frac{2 \pi}{T} \\
& >\frac{2 \pi}{T},
\end{aligned}
$$

that is

$$
\frac{m_{1}}{r_{1}^{3}}+\frac{m_{2}}{r_{2}^{3}}>\frac{4 \pi^{2}}{T^{2}} .
$$

By (3.18), we have

$$
\begin{aligned}
\delta^{2} f(O, \tilde{\varphi}) & =\int_{0}^{T}\left[\frac{4 \pi^{2}}{T^{2}} \cos ^{2} \frac{2 \pi}{T} t-\left(\frac{m_{1}}{r_{1}^{3}}+\frac{m_{2}}{r_{2}^{3}}\right) \sin ^{2} \frac{2 \pi}{T} t\right] d t \\
& =\frac{2 \pi^{2}}{T}-\left(\frac{m_{1}}{r_{1}^{3}}+\frac{m_{2}}{r_{2}^{3}}\right) \cdot \frac{T}{2} \\
& =\left[\frac{4 \pi^{2}}{T^{2}}-\left(\frac{m_{1}}{r_{1}^{3}}+\frac{m_{2}}{r_{2}^{3}}\right)\right] \cdot \frac{T}{2} \\
& <0,
\end{aligned}
$$

which implies $q(t)=(0,0,0)$ is not a local minimum for $f(q)$ on $\Lambda_{i}(i=1,2)$. Hence the minimizers of $f(q)$ on $\Lambda_{i}(i=1,2)$ are not always at the center of the masses, they must oscillate periodically on the vertical axis, that is, the minimizers are not always co-planar, therefore, we get nonplanar periodic solutions.

Combined with Lemma 2.4, the proof is completed.

\section{Proof of Theorem 1.2}

In this section, we consider the spatial circular restricted 4-body problem with a zero mass point moving on the vertical axis of the moving plane for three mass points with arbitrary positive masses $m_{1}, m_{2}, m_{3}$ in a Lagrange configuration. Suppose there exist $\theta_{1}, \theta_{2}, \theta_{3} \in[0,2 \pi)$ such that the planar circular orbits are

$$
\begin{aligned}
& q_{1}(t)=\left(r_{1} \cos \left(\frac{2 \pi}{T} t+\theta_{1}\right), r_{1} \sin \left(\frac{2 \pi}{T} t+\theta_{1}\right), 0\right), \\
& q_{2}(t)=\left(r_{2} \cos \left(\frac{2 \pi}{T} t+\theta_{2}\right), r_{2} \sin \left(\frac{2 \pi}{T} t+\theta_{2}\right), 0\right),
\end{aligned}
$$




$$
q_{3}(t)=\left(r_{3} \cos \left(\frac{2 \pi}{T} t+\theta_{3}\right), r_{3} \sin \left(\frac{2 \pi}{T} t+\theta_{3}\right), 0\right)
$$

here the radius $r_{1}, r_{2}, r_{3}$ are positive constants depending on $m_{i}(i=1,2,3)$ and $T$ (see Lemma 4.2). We also assume that

$$
m_{1} q_{1}(t)+m_{2} q_{2}(t)+m_{3} q_{3}(t)=0
$$

and

$$
\left|q_{i}-q_{j}\right|=l, \quad 1 \leq i \neq j \leq 3,
$$

where the constant $l>0$ depends on $m_{i}(i=1,2,3)$ and $T$ (see Lemma 4.1). We consider the Lagrangian functional of equation (1.3)

$$
\begin{aligned}
f(q) & =\int_{0}^{T}\left[\frac{1}{2}|\dot{q}|^{2}+\frac{m_{1}}{\left|q-q_{1}\right|}+\frac{m_{2}}{\left|q-q_{2}\right|}+\frac{m_{3}}{\left|q-q_{3}\right|}\right] d t \\
& =\int_{0}^{T}\left[\frac{1}{2}\left|z^{\prime}\right|^{2}+\frac{m_{1}}{\sqrt{r_{1}^{2}+z^{2}}}+\frac{m_{2}}{\sqrt{r_{2}^{2}+z^{2}}}+\frac{m_{3}}{\sqrt{r_{3}^{2}+z^{2}}}\right] d t \triangleq f(z)
\end{aligned}
$$

on $\Lambda_{i}, i=1,2$.

In order to get Theorem 1.2, we firstly prove Lemmas 4.1 and 4.2 as follows.

Lemma 4.1 Let $M=m_{1}+m_{2}+m_{3}$, we have $l=\sqrt[3]{\frac{M T^{2}}{4 \pi^{2}}}$.

Proof It follows from (1.1) and (1.2) that

$$
\ddot{q}_{1}=m_{2} \frac{q_{2}-q_{1}}{\left|q_{2}-q_{1}\right|^{3}}+m_{3} \frac{q_{3}-q_{1}}{\left|q_{3}-q_{1}\right|^{3}} .
$$

Then by (4.1), (4.4), and (4.5), we obtain

$$
\begin{aligned}
-\frac{4 \pi^{2}}{T^{2}} q_{1} & =\frac{1}{l^{3}}\left(m_{2} q_{2}+m_{3} q_{3}-m_{2} q_{1}-m_{3} q_{1}\right) \\
& =\frac{1}{l^{3}}\left(-m_{1} q_{1}-m_{2} q_{1}-m_{3} q_{1}\right)
\end{aligned}
$$

which implies

$$
l^{3}=\frac{M T^{2}}{4 \pi^{2}}
$$

that is,

$$
l=\sqrt[3]{\frac{M T^{2}}{4 \pi^{2}}} .
$$

Lemma 4.2 The radius $r_{1}, r_{2}, r_{3}$ of the planar circular orbits for the masses $m_{1}, m_{2}, m_{3}$ are

$$
r_{1}=\frac{\sqrt{m_{2}^{2}+m_{2} m_{3}+m_{3}^{2}}}{M} l
$$




$$
\begin{aligned}
& r_{2}=\frac{\sqrt{m_{1}^{2}+m_{1} m_{3}+m_{3}^{2}}}{M} l \\
& r_{3}=\frac{\sqrt{m_{1}^{2}+m_{1} m_{2}+m_{2}^{2}}}{M} l
\end{aligned}
$$

Proof Choose the geometrical center of the initial configuration $\left(q_{1}(0), q_{2}(0), q_{3}(0)\right)$ as the origin of the coordinate $(x, y, z)$. Without loss of generality, by (4.5), we may suppose the location coordinates of $q_{1}(0), q_{2}(0), q_{3}(0)$ are $A_{1}\left(\frac{\sqrt{3} l}{3}, 0,0\right), A_{2}\left(-\frac{\sqrt{3} l}{6}, \frac{l}{2}, 0\right)$, $A_{3}\left(-\frac{\sqrt{3} l}{6},-\frac{l}{2}, 0\right)$. Then we can get the coordinate of the center of masses $m_{1}, m_{2}, m_{3}$ is $C\left(\frac{\frac{\sqrt{3}}{3} m_{1} l-\frac{\sqrt{3}}{6} m_{2} l-\frac{\sqrt{3}}{6} m_{3} l}{M}, \frac{\frac{m_{2}}{2} l-\frac{m_{3}}{2} l}{M}, 0\right)$. To make sure assumption (4.4) holds, we introduce the new coordinates

$$
\left\{\begin{array}{l}
X=x-\frac{\frac{\sqrt{3}}{3} m_{1} l-\frac{\sqrt{3}}{6} m_{2} l-\frac{\sqrt{3}}{6} m_{3} l}{M}, \\
Y=y-\frac{\frac{m_{2}}{2} l-\frac{m_{3}}{2} l}{M}, \\
Z=z .
\end{array}\right.
$$

Hence in the new coordinates $(X, Y, Z)$, the location coordinates of $q_{1}(0), q_{2}(0), q_{3}(0)$ are $A_{1}\left(\frac{\frac{\sqrt{3}}{2} m_{2} l+\frac{\sqrt{3}}{2} m_{3} l}{M}, \frac{-\frac{m_{2}}{2} l+\frac{m_{3}}{2} l}{M}, 0\right), A_{2}\left(-\frac{\frac{\sqrt{3}}{2} m_{1} l}{M}, \frac{\frac{m_{1}}{2} l+m_{3} l}{M}, 0\right), A_{3}\left(-\frac{\frac{\sqrt{3}}{2} m_{1} l}{M},-\frac{\frac{m_{1}}{2} l+m_{2} l}{M}, 0\right)$ and the center of the masses $m_{1}, m_{2}, m_{3}$ are at the origin $O(0,0,0)$. Then compared with (4.1)-(4.3), we have

$$
\begin{aligned}
& r_{1}=\left|A_{1} O\right|=\frac{\sqrt{m_{2}^{2}+m_{2} m_{3}+m_{3}^{2}}}{M} l, \\
& r_{2}=\left|A_{2} O\right|=\frac{\sqrt{m_{1}^{2}+m_{1} m_{3}+m_{3}^{2}}}{M} l, \\
& r_{3}=\left|A_{3} O\right|=\frac{\sqrt{m_{1}^{2}+m_{1} m_{2}+m_{2}^{2}}}{M} l
\end{aligned}
$$

and

$$
\begin{aligned}
& \sin \theta_{1}=\frac{-m_{2}+m_{3}}{2 \sqrt{m_{2}^{2}+m_{2} m_{3}+m_{3}^{2}}}, \quad \cos \theta_{1}=\frac{\sqrt{3}\left(m_{2}+m_{3}\right)}{2 \sqrt{m_{2}^{2}+m_{2} m_{3}+m_{3}^{2}}}, \\
& \sin \theta_{2}=\frac{m_{1}+2 m_{3}}{2 \sqrt{m_{1}^{2}+m_{1} m_{3}+m_{3}^{2}}}, \quad \cos \theta_{2}=-\frac{\sqrt{3} m_{1}}{2 \sqrt{m_{1}^{2}+m_{1} m_{3}+m_{3}^{2}}}, \\
& \sin \theta_{3}=-\frac{m_{1}+2 m_{2}}{2 \sqrt{m_{1}^{2}+m_{1} m_{2}+m_{2}^{2}}}, \\
& \cos \theta_{3}=-\frac{\sqrt{3} m_{1}}{2 \sqrt{m_{1}^{2}+m_{1} m_{2}+m_{2}^{2}}} .
\end{aligned}
$$

Proof of Theorem 1.2 Clearly, $q(t)=(0,0,0)$ is a critical point of $f(q)$ on $\bar{\Lambda}_{i}=\Lambda_{i}(i=1,2)$. The second variation of (4.6) in the sphere neighborhood of $q(t)=(0,0,0)$ (coordinate origin $O$ ) is given by

$$
\delta^{2} f(O, \varphi)=\int_{0}^{T}\left(\varphi_{3}^{\prime 2}-\left(\frac{m_{1}}{r_{1}^{3}}+\frac{m_{2}}{r_{2}^{3}}+\frac{m_{3}}{r_{3}^{3}}\right) \varphi_{3}^{2}\right) d t, \quad \forall \varphi=\left(0,0, \varphi_{3}\right) \in \Lambda_{i}, i=1,2 .
$$


Let

$$
\tilde{\varphi}(t)=\left(0,0, \sin \frac{2 \pi}{T} t\right)
$$

and

$$
\begin{aligned}
& A=\frac{\sqrt{m_{2}^{2}+m_{2} m_{3}+m_{3}^{2}}}{M}, \\
& B=\frac{\sqrt{m_{1}^{2}+m_{1} m_{3}+m_{3}^{2}}}{M}, \\
& C=\frac{\sqrt{m_{1}^{2}+m_{1} m_{2}+m_{2}^{2}}}{M} .
\end{aligned}
$$

Obviously, $\tilde{\varphi} \in \Lambda_{i}(i=1,2)$ and

$$
\dot{\tilde{\varphi}}(t)=\left(0,0, \frac{2 \pi}{T} \cos \frac{2 \pi}{T} t\right) .
$$

It is easy to check that

$$
\begin{aligned}
& M^{2}>m_{1}^{2}+m_{1} m_{2}+m_{2}^{2}, \\
& M^{2}>m_{1}^{2}+m_{1} m_{3}+m_{3}^{2}, \\
& M^{2}>m_{2}^{2}+m_{2} m_{3}+m_{3}^{2},
\end{aligned}
$$

which implies

$$
\frac{m_{1}}{A^{3}}+\frac{m_{2}}{B^{3}}+\frac{m_{3}}{C^{3}}>m_{1}+m_{2}+m_{3}=M .
$$

Therefore

$$
\frac{\sqrt{M}}{\sqrt{\frac{m_{1}}{A^{3}}+\frac{m_{2}}{B^{3}}+\frac{m_{3}}{C^{3}}}}<1,
$$

that is,

$$
\frac{\sqrt{\frac{m_{1}}{A^{3}}+\frac{m_{2}}{B^{3}}+\frac{m_{3}}{C^{3}}}}{\sqrt{M}}>1 .
$$

It follows from (4.10)-(4.13) and (4.23) that

$$
\begin{aligned}
\sqrt{\frac{m_{1}}{r_{1}^{3}}+\frac{m_{2}}{r_{2}^{3}}+\frac{m_{3}}{r_{3}^{3}}} & =\sqrt{\frac{m_{1}}{A^{3}}+\frac{m_{2}}{B^{3}}+\frac{m_{3}}{C^{3}}} \sqrt{\frac{1}{l^{3}}} \\
& =\sqrt{\frac{m_{1}}{A^{3}}+\frac{m_{2}}{B^{3}}+\frac{m_{3}}{C^{3}}} \cdot \frac{2 \pi}{\sqrt{M} T} \\
& >\frac{2 \pi}{T}
\end{aligned}
$$


that is,

$$
\frac{m_{1}}{r_{1}^{3}}+\frac{m_{2}}{r_{2}^{3}}+\frac{m_{3}}{r_{3}^{3}}>\frac{4 \pi^{2}}{T^{2}}
$$

By (4.24), we have

$$
\begin{aligned}
\delta^{2} f(O, \tilde{\varphi}) & =\int_{0}^{T}\left[\frac{4 \pi^{2}}{T^{2}} \cos ^{2} \frac{2 \pi}{T} t-\left(\frac{m_{1}}{r_{1}^{3}}+\frac{m_{2}}{r_{2}^{3}}+\frac{m_{3}}{r_{3}^{3}}\right) \sin ^{2} \frac{2 \pi}{T} t\right] d t \\
& =\frac{2 \pi^{2}}{T}-\left(\frac{m_{1}}{r_{1}^{3}}+\frac{m_{2}}{r_{2}^{3}}+\frac{m_{3}}{r_{3}^{3}}\right) \cdot \frac{T}{2} \\
& =\left[\frac{4 \pi^{2}}{T^{2}}-\left(\frac{m_{1}}{r_{1}^{3}}+\frac{m_{2}}{r_{2}^{3}}+\frac{m_{3}}{r_{3}^{3}}\right)\right] \cdot \frac{T}{2} \\
& <0,
\end{aligned}
$$

which implies $q(t)=(0,0,0)$ is not a local minimum for $f(q)$ on $\Lambda_{i}(i=1,2)$. Hence the minimizers of $f(q)$ on $\Lambda_{i}(i=1,2)$ are not always at the center of the masses, they must oscillate periodically on the vertical axis, that is, the minimizers are not always co-planar, therefore, we get nonplanar periodic solutions.

Combined with Lemma 2.4, the proof is completed.

\section{Competing interests}

The authors declare that they have no competing interests.

\section{Authors' contributions}

All authors contributed equally to the manuscript, and they read and approved the final draft.

\section{Author details}

${ }^{1}$ School of Mathematics and Statistics, Henan University of Science and Technology, Luoyang, 471023, People's Republic of China. ${ }^{2}$ Yangtze Center of Mathematics, College of Mathematics, Sichuan University, Chengdu, 610064, People's Republic of China.

\section{Acknowledgements}

The authors would like to thank Donglun Wu for some helpful discussions. The authors also would like to thank the referees for valuable suggestions.

Received: 1 January 2015 Accepted: 4 May 2015 Published online: 28 May 2015

\section{References}

1. Li, F, Zhang, S, Zhao, X: The characterization of the variational minimizers for spatial restricted $N+1$-body problems. Abstr. Appl. Anal. 2013, Article ID 845795 (2013)

2. Gelfand, I, Fomin, S: Calculus of Variations. Nauka, Moscow (1965). English edition: Prentice Hall, Englewood Cliffs

3. Struwe, M: Variational Methods, 3rd edn. Springer, Berlin (1990)

4. McGehee, R: Parabolic Orbits of the Restricted Three-Body Problem. Academic Press, New York (1973)

5. Mathlouthi, S: Periodic orbits of the restricted three-body problem. Trans. Am. Math. Soc. 350, 2265-2276 (1998)

6. Zhang, SQ: Variational minimizing parabolic and hyperbolic orbits for the restricted 3-body problems. Sci. China Math. 55, 721-725 (2012)

7. Sitnikov, K: Existence of oscillating motions for the three-body problem. Dokl. Akad. Nauk. USSR 133, 303-306 (1960)

8. Mawhin, J, Willem, M: Critical Point Theory and Hamiltonian Systems. Applied Mathematical Sciences, vol. 74. Springer, New York (1989)

9. Palais, R: The principle of symmetric criticality. Commun. Math. Phys. 69, 19-30 (1979) 